P129 WHAT IS THE PATIENT'S PREFERENCE: TIOTROPIUM MONOTHERAPY OR FIXED-DOSE INDACATEROL/ GLYCOPYRRONIUM COMBINATION THERAPY? - THE FAVOUR STUDY

P Kardos, I Hagedorn. Allergy, Respiratory and Sleep Medicine, Red Cross Maingau Hospital, Frankfurt, Germany

\subsection{6/thoraxjnl-2015-207770.266}

Introduction and objectives Shared decision-making for drug and inhaler-device use improves adherence and outcomes in COPD. Physicians are encouraged to involve patient's opinion in choosing drugs and inhalers. It remains unclear, however, whether the superior effect on FEV1 shown for the fixed-dose combination of indacaterol/glycopyrronium (IND/GLY) compared to tiotropium (TIO) translates into medication preference by patients.

The present study was conducted to evaluate the overall preference for IND/GLY and TIO respectively, in patients who are still symptomatic under a TIO treatment.

Method This multicenter, cross-over, open-label study randomised COPD patients with moderate to severe airflow limitation and a CAT score of $\geq 10$ to either receive 4 weeks o.d. IND/GLY $(110 / 50 \mu \mathrm{g})$ followed by 4 weeks o.d. TIO $(18 \mu \mathrm{g})$ or vice versa in a 1:1 ratio. To determine patient's treatment preference and satisfaction as secondary and explorative objectives, respectively, several validated and new questionnaires were used. As primary endpoint, FEV1 $1 \mathrm{~h}$ post-inhalation after 4 weeks was investigated.

Results Of 88 patients (mean age, 65 years; post-bronchodilator FEV1, 57.7\% predicted; mean CAT score, 17.6) randomised, 87 patients completed the study. After 4 weeks treatment, $1 \mathrm{~h}$ postinhalation FEV1 was significantly higher with IND/GLY compared to TIO (LSM difference, $81 \mathrm{ml} ; \mathrm{p}=0.0017$ ). Importantly, a higher proportion of patients preferred IND/GLY (69.4\%) over TIO (30.6\%) at the end of the study. Reduction of dyspnea was mentioned as an important or very important reason for favouring IND/GLY by $91.5 \%$ of the patients.

Conclusion This study indicated that beyond FEV1, patients reported outcomes improve with the dual bronchodilator IND/
GLY compared to TIO monotherapy. Further studies are needed to investigate how the favoured treatment option translates into improved adherence and long term treatment outcomes.

\section{P130 EFFECTIVENESS AND SAFETY OF INITIATING TREATMENT WITH FLUTICASONE/SALMETEROL VIA MDI VERSUS DPI IN COPD}

${ }^{1} \mathrm{R}$ Jones, ${ }^{2} \mathrm{~J}$ Martin, ${ }^{3} \mathrm{~V}$ Thomas, ${ }^{4} \mathrm{D}$ Skinner, ${ }^{5} \mathrm{~J}$ Marshall, ${ }^{6} \mathrm{D}$ Price. ${ }^{1}$ Centre for Clinical Trials and Health Research, Plymouth University, Plymouth, UK; ${ }^{2}$ Research in Real Life, Cambridge, UK; ${ }^{3}$ Cambridge Research Support, Cambridge, UK; ${ }^{4}$ Optimum Patient Care, Cambridge, UK; ${ }^{5}$ Mundipharma International Limited, Cambridge, UK; ${ }^{6}$ Observational and Pragmatic Research Institute, Singapore, Singapore

\subsection{6/thoraxjnl-2015-207770.267}

Introduction and objectives Fluticasone propionate/salmeterol (FP/SAL), can be delivered by metered-dose inhaler (MDI) or dry powder inhaler (DPI). The choice of device may affect adherence to and effectiveness of treatment. Although only 1000 $\mathrm{mcg} /$ day DPI is licensed for the treatment of COPD in the UK, the MDI and lower doses are regularly used in real-world practice. The aim of this study was to compare the effectiveness and safety of FP/SAL MDI or DPI at two doses $(500$ and $1000 \mathrm{mcg} /$ day) in COPD patients.

Methods Historical, matched cohort study using the Optimum Patient Care Research Database in patients with COPD, aged $\geq 35$ years and initiating with FP/SAL via either MDI or DPI. Conditional Poisson regression and conditional logistic regression were used respectively to compare the rate of moderate/ severe COPD exacerbations and the odds of diagnosis of pneumonia and diabetes mellitus (including anti-diabetic drug prescriptions) between MDI and DPI during one year outcome period. Models were adjusted for the respective baseline values of the outcome variable of interest where possible. Addition of LAMA therapy during the outcome period was compared using conditional logistic regression.

Results 472 and 1172 patients initiated on FP/SAL at $500 \mathrm{mcg} /$ day and $1000 \mathrm{mcg} / \mathrm{day}$, respectively. The rate of moderate/severe COPD exacerbations was significantly lower for patients prescribed

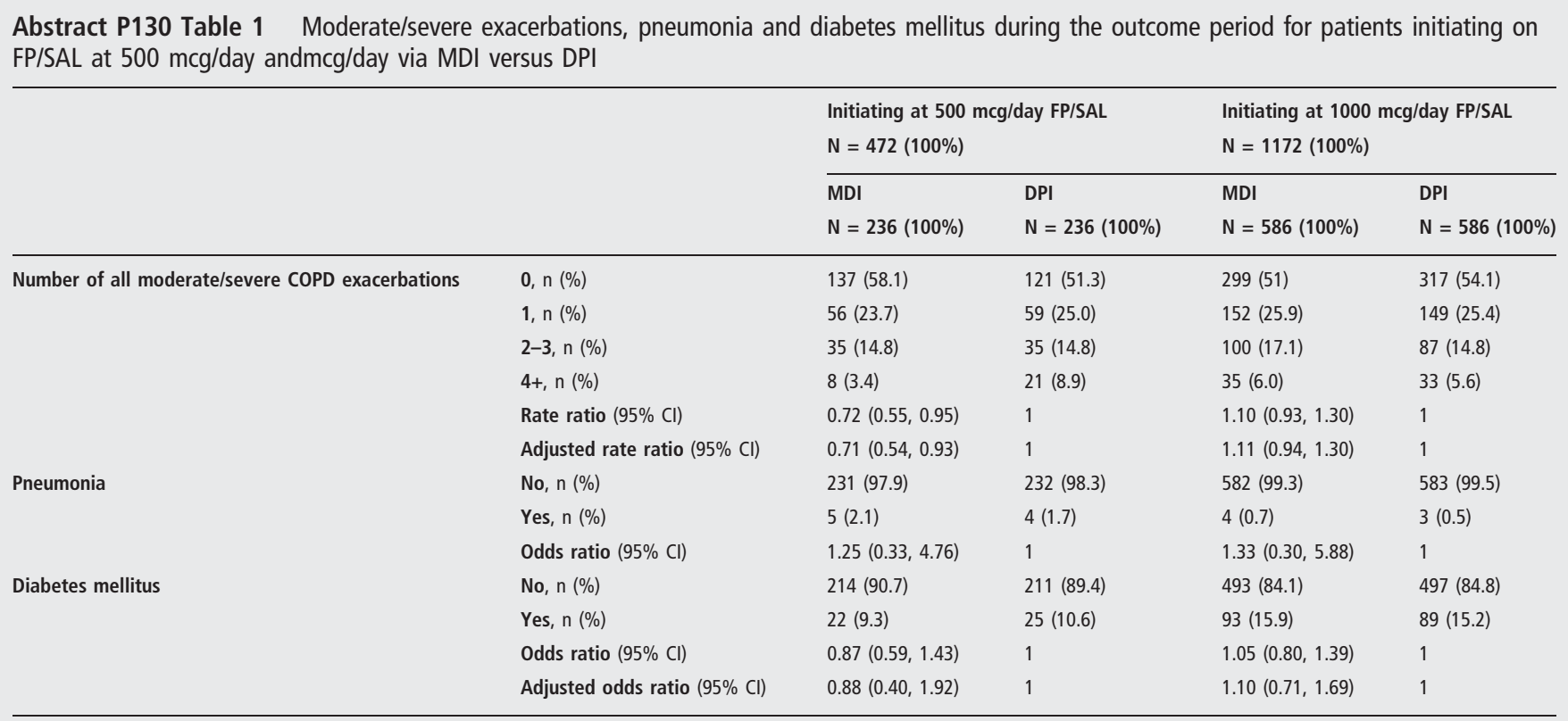

